\title{
Strong field gravitational lensing in the deformed Hořava-Lifshitz black hole
}

\author{
Songbai Chen* and Jiliang Jingt \\ Institute of Physics and Department of Physics, \\ Hunan Normal University, Changsha, Hunan 410081, P. R. China \\ Key Laboratory of Low Dimensional Quantum Structures and Quantum \\ Control (Hunan Normal University), Ministry of Education, P. R. China.
}

\begin{abstract}
Adopting the strong field limit approach, we studied the properties of strong field gravitational lensing in the deformed Hořava-Lifshitz black hole and obtained the angular position and magnification of the relativistic images. Supposing that the gravitational field of the supermassive central object of the galaxy described by this metric, we estimated the numerical values of the coefficients and observables for gravitational lensing in the strong field limit. Comparing with the Reissner-Norström black hole, we find that with the increase of parameter $\alpha$, the angular position $\theta_{\infty}$ decreases more slowly and $r_{m}$ more quickly, but angular separation $s$ increases more rapidly. This may offer a way to distinguish a deformed Hořava-Lifshitz black hole from a Reissner-Norström black hole by the astronomical instruments in the future.
\end{abstract}

PACS numbers: 04.70.-s, 95.30.Sf, 97.60.Lf

*Electronic address: csb3752@163.com

$\dagger$ Electronic address: jljing@hunnu.edu.cn 


\section{INTRODUCTION}

The general theory of relativity tells us that photons would be deviated from their straight path when they pass close to a compact and massive body. The phenomena resulting from the deflection of light rays in a gravitational field are called as gravitational lensing and the object causing a detectable deflection is usually named as a gravitational lens. Like a natural and large telescope, gravitational lensing can provide us the information about the distant stars which are otherwise too dim to be observed. Moreover, it can help us to detect the exotic objects (such as cosmic strings) in the universe as well as to verify alternative theories of gravity. Most of the theories of gravitational lensing have been developed in the weak field approximation [1]-[3] in which one only keeps the first non null term in the expansion of the deflection angle. In general, it is enough for us to investigate the properties of gravitational lensing by ordinary stars and galaxies. However, when the lens is a compact object with a photon sphere (such as black hole), a strong field treatment of gravitational lensing [4, 5, 6, 7, 8, 9] is need instead because that in this situation photons passing close to the photon sphere have large deflection angles and the weak field approximation is no valid any more. Virbhadra and Ellis [6] find that near the line connecting the source and the lens, an observer would detect two infinite sets of faint relativistic images on each side of the black hole which are produced by photons that make complete loops around the black hole before reaching the observer. These relativistic images could provide a profound verification of alternative theories of gravity in their strong field regime. Thus, the study of the strong field gravitational lensing by black holes in the different spacetimes becomes appealing recent years.

On the basis of the Virbhadra-Ellis lens equation [7, 8], Bozza [10] devised an analytical method for calculating the deflection angles for the light rays propagating close to the Schwarzschild black hole and showed that there exists a logarithmic divergence of the deflection angles at photon sphere. Later Bozza's technique was extended to other static spacetimes. For example, Eiroa et al [11]-[14] have studied the gravitational lensing due to the Reissner-Nordström black hole, braneworld black hole and Einstein-Born-Infeld black hole. Bozza [15] extended the analytical method of lensing for a general class of static and spherically symmetric spacetimes and showed that the logarithmic divergence of the deflection angle at photon sphere is a common feature for such spacetimes. Moreover, he [16]-18] has also studied the gravitational lensing by a spinning black hole. Bhadra et al [19][20] have considered the gravitational lensing by the Gibbons-Maeda-GarfinkleHorowitz-Strominger charged black hole and the black hole in the Brans-Dicke theory. Konoplya [21] has 
studied the corrections to the deflection angle and time delay in the background of a black hole immersed in a uniform magnetic field. Majumdar [22] has investigated the gravitational lensing in the dilaton-de Sitter black hole spacetimes. Perlick [23] has obtained an exact gravitational lens equation in a spherically symmetric and static spacetime and used it to study lensing by a Barriola-Vilenkin monopole black hole. Gyulchev [24] has studied the features of light propagation close to the equatorial plane of the rotating dilaton-axion black hole spacetime and obtained that there exists a significant dilaton-axion effect present on the gravitational lensing observables in the strong field limit. These results are very useful for us to verify the validity of gravity theories through the astronomical observation of the relativistic images in the universe.

Recently, Hořava 25] proposes a renormalizable four-dimensional theory of gravity, which admits the Lifshitz scale-invariance in time and space rather than Lorentz invariant theory of gravity in $3+1$ dimensions. Thereafter, the Hořava-Lifshitz gravity theory has been intensively investigated in 26, 27, 28, 29, 30, 31, 32, 33, 34, 35] and its cosmological applications have been studied in [36, 37, 38, 39, 40, 41, 42. Some static spherically symmetric black hole solutions have been found in Hořava-Lifshitz theory [43, 44, 45, 46, 47, 48] and the associated thermodynamic properties with those black hole solutions have been investigated in [49, 50, 51, 52]. The quasinormal modes of the massless scalar perturbations [53, 54] and the gravity lens in the weak field limit [54] have been studied in the deformed Hořava-Lifshitz black hole spacetime. Since the weak field limit takes the first order deviation from Minkowski spacetime, it is necessary to study the gravity lens in the strong field limit in the black hole spacetime because that it starts from complete capture of the photon and takes the leading order in the divergence of the deflection angle. The main purpose of this paper is to study the gravity lens in the strong field limit in the deformed Hořava-Lifshitz black hole spacetime [44].

The plan of our paper is organized as follows. In Sec.II we adopt to Bozza's method and obtain the deflection angles for light rays propagating in the deformed Hořava-Lifshitz black hole. In Sec.III we suppose that the gravitational field of the supermassive black hole at the centre of our galaxy can be described by this metric and then obtain the numerical results for the observational gravitational lensing parameters defined in Sec.II. Then, we make a comparison between the properties of gravitational lensing in the deformed Hořava-Lifshitz and Reissner-Norström metrics. At last, we present a summary. 


\section{DEFLECTION ANGLE IN THE DEFORMED HOŘAVA-LIFSHITZ BLACK HOLE}

In the Horava-Lifshitz gravity, the deformed action in the limit $\Lambda_{W} \rightarrow 0$ can be described by [44]

$$
\begin{aligned}
S_{H L} & =\int d t d^{3} x\left(\mathcal{L}_{0}+\tilde{\mathcal{L}}_{1}\right), \\
\mathcal{L}_{0} & =\sqrt{g} N\left\{\frac{2}{\kappa^{2}}\left(K_{i j} K^{i j}-\lambda K^{2}\right)+\frac{\kappa^{2} \mu^{2}\left(\Lambda_{W} R-3 \Lambda_{W}^{2}\right)}{8(1-3 \lambda)}\right\}, \\
\tilde{\mathcal{L}}_{1} & =\sqrt{g} N\left\{\frac{\kappa^{2} \mu^{2}(1-4 \lambda)}{32(1-3 \lambda)} R^{2}-\frac{\kappa^{2}}{2 w^{4}}\left(C_{i j}-\frac{\mu w^{2}}{2} R_{i j}\right)\left(C^{i j}-\frac{\mu w^{2}}{2} R^{i j}\right)+\mu^{4} R\right\} .
\end{aligned}
$$

Here $w, \lambda, \mu$ and $\kappa$ are the parameters in the Horava-Lifshitz theory. $K_{i j}$ is extrinsic curvature

$$
K_{i j}=\frac{1}{2 N}\left(\partial_{t} g_{i j}-\nabla_{i} N_{j}-\nabla_{j} N_{i}\right) .
$$

and $C_{i j}$ is the Cotton tensor

$$
C^{i j}=\epsilon^{i k \ell} \nabla_{k}\left(R_{\ell}^{j}-\frac{1}{4} R \delta_{\ell}^{j}\right)=\epsilon^{i k \ell} \nabla_{k} R_{\ell}^{j}-\frac{1}{4} \epsilon^{i k j} \partial_{k} R
$$

respectively. For $\lambda=1$, there exist a static and asymptotically flat black hole solution which has a form [44]

$$
d s^{2}=-f(r) d t^{2}+\frac{d r^{2}}{f(r)}+r^{2}\left(d \theta^{2}+\sin ^{2} \theta d \phi^{2}\right)
$$

and

$$
f(r)=\frac{2\left(r^{2}-2 M r+\alpha\right)}{r^{2}+2 \alpha+\sqrt{r^{4}+8 \alpha M r}}
$$

where $\alpha=1 /(2 w)$ and $M$ is an integration constant related to the mass. Obviously, it returns the Schwarzschild spacetime as the parameter $\alpha=0$. When the mass $M=0$, it is corresponding to the Minkowski spacetime. Although the metric of this black hole behaviors as that of Reissner-Norström black hole and the event horizons are given by

$$
r_{ \pm}=M \pm \sqrt{M^{2}-\alpha}
$$

there exist a distinct difference between them is that the denominator of $f(r)$ in the deformed Hořava-Lifshitz black hole metric is no longer equal to $r$, which will make a great deal influence on gravitational lensing in the strong field limit.

As in [7, 8, 15], we just consider that both the observer and the source lie in the equatorial plane in in the deformed Hořava-Lifshitz black hole (2.6) and the whole trajectory of the photon is limited on the same plane. With the conditions that $\theta=\frac{\pi}{2}$ and $2 M=1$, the metric (2.6) can be rewritten as

$$
d s^{2}=-A(r) d t^{2}+B(r) d r^{2}+C(r) d \phi^{2}
$$


with

$$
A(r)=f(r), \quad B(r)=1 / f(r), \quad C(r)=r^{2} .
$$

The deflection angle for the photon coming from infinite can be expressed as

$$
\alpha\left(r_{0}\right)=I\left(r_{0}\right)-\pi,
$$

where $r_{0}$ is the closest approach distance and $I\left(r_{0}\right)$ is $[7,8]$

$$
I\left(r_{0}\right)=2 \int_{r_{0}}^{\infty} \frac{\sqrt{B(r)} d r}{\sqrt{C(r)} \sqrt{\frac{C(r) A\left(r_{0}\right)}{C\left(r_{0}\right) A(r)}-1}} .
$$

It is easy to obtain that as parameter $r_{0}$ decrease the deflection angle increase. At certain a point, the deflection angle will become $2 \pi$, it means that the light ray will make a complete loop around the compact object before reaching the observer. When $r_{0}$ is equal to radius of the photon sphere, the deflection angle diverges and the photon is captured.

The photon sphere equation is given by [7, 8]

$$
\frac{C^{\prime}(r)}{C(r)}=\frac{A^{\prime}(r)}{A(r)}
$$

which admits at least one positive solution and then the largest real root of Eq.(2.13) is defined as the radius of the photon sphere. In the deformed Hořava-Lifshitz black hole metric, the radius of the photon sphere can be given explicitly by

$$
r_{p s}=\frac{3+\left(\sqrt{256 \alpha^{2}-27}-16 \alpha^{2}\right)^{2 / 3}}{2\left(\sqrt{256 \alpha^{2}-27}-16 \alpha^{2}\right)^{1 / 3}} .
$$

When $\alpha=0$, it can recovers that in the Schwarzschild black hole spacetime in which $r_{p s}=1.5$. However, it is quite a different from that in the Reissner-Norström black hole spacetime, which implies that there exist some distinct effects of the Hořava-Lifshitz parameter $\alpha$ on gravitational lensing in the strong field limit. Following the method developed by Bozza [15], we define a variable

$$
z=1-\frac{r_{0}}{r}
$$

and obtain

$$
I\left(r_{0}\right)=\int_{0}^{1} R\left(z, r_{0}\right) f\left(z, r_{0}\right) d z,
$$

where

$$
R\left(z, r_{0}\right)=\frac{2 r^{2} \sqrt{A(r) B(r) C\left(r_{0}\right)}}{r_{0} C(r)}=2,
$$




$$
f\left(z, r_{0}\right)=\frac{1}{\sqrt{A\left(r_{0}\right)-A(r) C\left(r_{0}\right) / C(r)}} .
$$

The function $R\left(z, r_{0}\right)$ is regular for all values of $z$ and $r_{0}$. However, $f\left(z, r_{0}\right)$ diverges as $z$ tends to zero. Thus, we split the integral (2.16) into two parts

$$
\begin{aligned}
I_{D}\left(r_{0}\right) & =\int_{0}^{1} R\left(0, r_{p s}\right) f_{0}\left(z, r_{0}\right) d z \\
I_{R}\left(r_{0}\right) & =\int_{0}^{1}\left[R\left(z, r_{0}\right) f\left(z, r_{0}\right)-R\left(0, r_{p s}\right) f_{0}\left(z, r_{0}\right)\right] d z,
\end{aligned}
$$

where $I_{D}\left(r_{0}\right)$ and $I_{R}\left(r_{0}\right)$ denote the divergent and regular parts in the integral (2.16), respectively. To find the order of divergence of the integrand, we expand the argument of the square root in $f\left(z, r_{0}\right)$ to the second order in $z$ and obtain the function $f_{0}\left(z, r_{0}\right)$ :

$$
f_{0}\left(z, r_{0}\right)=\frac{1}{\sqrt{p\left(r_{0}\right) z+q\left(r_{0}\right) z^{2}}}
$$

where

$$
\begin{aligned}
& p\left(r_{0}\right)=2-\frac{3 r_{0}}{\sqrt{r_{0}^{4}+4 \alpha r_{0}}}, \\
& q\left(r_{0}\right)=\frac{3 r_{0}\left(r_{0}^{3}+\alpha\right)}{\left(r_{0}^{3}+4 \alpha\right) \sqrt{r_{0}^{4}+4 \alpha r_{0}}}-1 .
\end{aligned}
$$

When $r_{0}$ is equal to the radius of photon sphere $r_{p s}$, the coefficient $p\left(r_{0}\right)$ vanishes and the leading term of the

divergence in $f_{0}\left(z, r_{0}\right)$ is $z^{-1}$, thus the integral (2.16) diverges logarithmically. Close to the divergence, Bozza 15] found that the deflection angle can be expanded in the form

$$
\alpha(\theta)=-\bar{a} \log \left(\frac{\theta D_{O L}}{u_{p s}}-1\right)+\bar{b}+O\left(u-u_{p s}\right)
$$

where

$$
\begin{aligned}
\bar{a} & =\frac{1}{\sqrt{q\left(r_{p s}\right)}}, \\
\bar{b} & =-\pi+b_{R}+\bar{a} \log \frac{r_{p s}^{2}\left[C^{\prime \prime}\left(r_{p s}\right) A\left(r_{p s}\right)-C\left(r_{p s}\right) A^{\prime \prime}\left(r_{p s}\right)\right]}{u_{p s} \sqrt{A^{3}\left(r_{p s}\right) C\left(r_{p s}\right)}} \\
b_{R} & =I_{R}\left(r_{p s}\right), \quad u_{p s}=\frac{r_{p s}}{\sqrt{A\left(r_{p s}\right)}}
\end{aligned}
$$

$D_{O L}$ denotes the distance between observer and gravitational lens, $\bar{a}$ and $\bar{b}$ are so-called the strong field limit coefficients which depend on the metric functions evaluated at $r_{p s}$. In general, the coefficient $b_{R}$ can not be calculated analytically and need to be evaluated numerically. Here we expand the integrand in (2.19) in powers of $\alpha$ as in [15], we can get

$$
b_{R}=b_{R, 0}+b_{R, 1} \alpha+O(\alpha)^{2},
$$



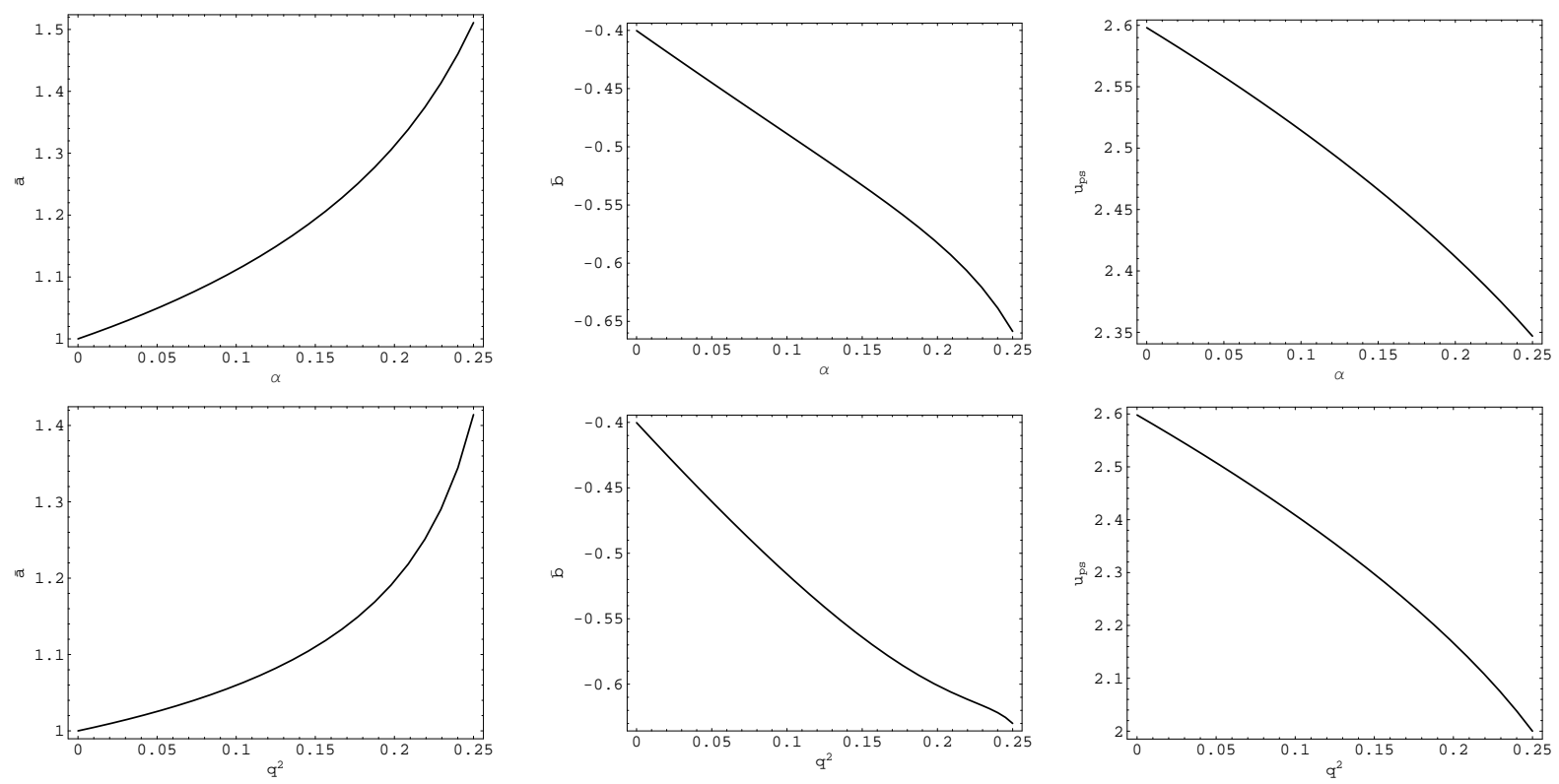

FIG. 1: Variation of the coefficients of the strong field limit $\bar{a}, \bar{b}$ and the minimum impact parameter $u_{p s}$ with parameter $\alpha$ in the deformed Hořava-Lifshitz black hole spacetime (in the upper row) and with $q^{2}$ in the Reissner-Norström black hole (in the lower row).

and evaluate the single coefficients $b_{R, 0}$ and $b_{R, 1} . b_{R, 0}$ is the value of the coefficient for a Schwarzschild black hole and $b_{R, 1}$ is the correction from the Hořava-Lifshitz parameter $\alpha$,

$$
b_{R, 1}=\frac{16}{45}[-13+4 \sqrt{3}+10 \log (3-\sqrt{3})]=-1.3148
$$

which is larger than that of the Reissner-Norström black hole where $b_{R, 1}=-1.5939$ [15]. Figures (1) tell us that with the increase of $\alpha$ increases the coefficient $\bar{a}$ increase, but both of the minimum impact parameter $u_{p s}$ and another coefficient $\bar{b}$ increases, which is similar to that in in the Reissner-Norström black hole metric. However, as shown in Fig. (1), in the deformed Hořava-Lifshitz black hole, $\bar{a}$ increases more quickly, both of $\bar{b}$ and $u_{p s}$ decrease more slowly. This means that in principle we can distinguish a deformed Hořava-Lifshitz black hole from a Reissner-Nordstrom black hole by using strong field gravitational lensing.

Considering the source, lens and observer are highly aligned, the lens equation in strong gravitational lensing can be written as [10]

$$
\beta=\theta-\frac{D_{L S}}{D_{O S}} \Delta \alpha_{n},
$$

where $D_{L S}$ is the distance between the lens and the source, $D_{O S}=D_{L S}+D_{O L}, \beta$ is the angular separation between the source and the lens, $\theta$ is the angular separation between the imagine and the lens, $\Delta \alpha_{n}=\alpha-2 n \pi$ is the offset of deflection angle and $n$ is an integer. The position of the $n$-th relativistic image can be approximated 
as

$$
\theta_{n}=\theta_{n}^{0}+\frac{u_{p s} e_{n}\left(\beta-\theta_{n}^{0}\right) D_{O S}}{\bar{a} D_{L S} D_{O L}}
$$

where

$$
e_{n}=e^{\frac{\bar{b}-2 n \pi}{\bar{a}}}
$$

$\theta_{n}^{0}$ are the image positions corresponding to $\alpha=2 n \pi$. The magnification of $n$-th relativistic image is given by

$$
\mu_{n}=\frac{u_{p s}^{2} e_{n}\left(1+e_{n}\right) D_{O S}}{\bar{a} \beta D_{L S} D_{O L}^{2}}
$$

If $\theta_{\infty}$ represents the asymptotic position of a set of images in the limit $n \rightarrow \infty$, the minimum impact parameter $u_{p s}$ can be simply obtained as

$$
u_{p s}=D_{O L} \theta_{\infty}
$$

In the simplest situation, we consider only that the outermost image $\theta_{1}$ is resolved as a single image and all the remaining ones are packed together at $\theta_{\infty}$. Then the angular separation between the first image and other ones can be expressed as

$$
s=\theta_{1}-\theta_{\infty}
$$

and the ratio of the flux from the first image and those from the all other images is given by

$$
\mathcal{R}=\frac{\mu_{1}}{\sum_{n=2}^{\infty} \mu_{n}} .
$$

For highly aligned source, lens and observer geometry, these observable can be simplified as

$$
\begin{aligned}
& s=\theta_{\infty} e^{\frac{\bar{b}-2 \pi}{\bar{a}}}, \\
& \mathcal{R}=e^{\frac{2 \pi}{\bar{a}}}
\end{aligned}
$$

The strong deflection limit coefficients $\bar{a}, \bar{b}$ and the minimum impact parameter $u_{p s}$ can be obtain through measuring $s, \mathcal{R}$ and $\theta_{\infty}$. Then, comparing their values with those predicted by the theoretical models, we can identify the nature of the black hole lens.

\section{NUMERICAL ESTIMATION OF OBSERVATIONAL GRAVITATIONAL LENSING PARAMETERS}

In this section, supposing that the gravitational field of the supermassive black hole at the galactic center of Milk Way can be described by the deformed Hořava-Lifshitz black hole spacetime, we estimate the numerical 
values for the coefficients and observables of gravitational lensing in the strong field limit, and then we study the effect of the metric parameter $\alpha$ on the gravitational lensing.
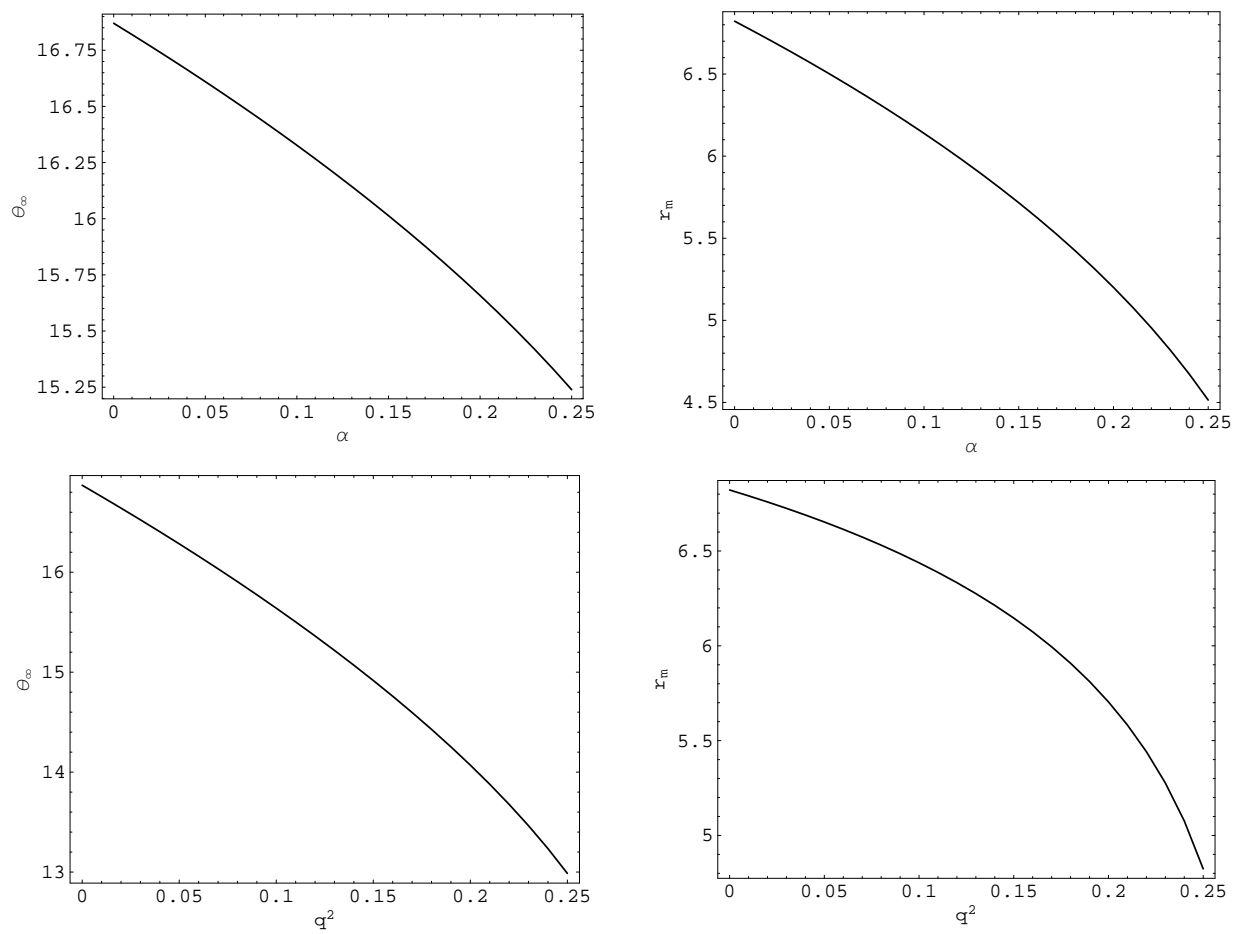

FIG. 2: Gravitational lensing by the Galactic center black hole. Variation of the values of the angular position $\theta_{\infty}$ and the relative magnitudes $r_{m}$ with parameter $\alpha$ in the deformed Hořava-Lifshitz black hole spacetime (in the upper row) and with $q^{2}$ in the Reissner-Norström black hole (in the lower row).

The mass of the central object of our Galaxy is estimated to be $2.8 \times 10^{6} M_{\odot}$ and its distance is around 7.6kpc. For different $\alpha$, the numerical value for the minimum impact parameter $u_{p s}$, the angular position of the relativistic images $\theta_{\infty}$, the angular separation $s$ and the relative magnification of the outermost relativistic image with the other relativistic images $r_{m}$ are listed in the table I. It is easy to obtain that our results reduce

\begin{tabular}{|c|c|c|c|c|c|c|}
\hline \hline$\alpha$ & $\theta_{\infty}(\mu$ arcsecs $)$ & $s$ ( $\mu$ arcsecs $)$ & $r_{m}$ (magnitudes) & $u_{p s} / R_{S}$ & $\bar{a}$ & $b$ \\
\hline 0 & 16.870 & 0.0211 & 6.8219 & 2.598 & 1.000 & -0.4002 \\
\hline 0.05 & 16.610 & 0.0273 & 6.5014 & 2.558 & 1.049 & -0.4450 \\
\hline 0.10 & 16.327 & 0.0368 & 6.1387 & 2.514 & 1.111 & -0.4888 \\
\hline 0.15 & 16.014 & 0.0530 & 5.7162 & 2.466 & 1.193 & -0.5331 \\
\hline 0.20 & 15.658 & 0.0835 & 5.2008 & 2.411 & 1.312 & -0.5826 \\
\hline 0.25 & 15.239 & 0.1541 & 4.5145 & 2.347 & 1.511 & -0.6586 \\
\hline \hline
\end{tabular}

TABLE I: Numerical estimation for main observables and the strong field limit coefficients for black hole at the center of our galaxy, which is supposed to be described by the deformed Hořava-Lifshitz black hole spacetime. $\alpha$ is the parameter of metric. $R_{s}$ is Schwarzschild radius. $r_{m}=2.5 \log \mathcal{R}$

to those in the Schwarzschild black hole sacetime as $\alpha=0$. Moreover, from the table I, we also find that as the parameter $\alpha$ increases, the minimum impact parameter $u_{p s}$, the angular position of the relativistic images 
$\theta_{\infty}$ and the relative magnitudes $r_{m}$ decrease, but the angular separation $s$ increases.

From Fig. (2), we find that in the deformed Hořava-Lifshitz black hole with the increase of parameter $\alpha$, the angular position $\theta_{\infty}$ decreases more slowly and $r_{m}$ more quickly, but angular separation $s$ increases more rapidly. This means that the bending angle and the relative magnification of the outermost relativistic image with the other relativistic images are smaller in the deformed Hořava-Lifshitz black hole. Our results also agree with that obtained by in the weak field limit [54]. In order to identify the nature of these two compact objects lensing, it is necessary for us to measure angular separation $s$ and the relative magnification $r_{m}$ in the astronomical observations. Tables I tell us that the resolution of two extremely faint images separated is $\sim 0.06 \mu$ arc sec, which is too small. However, with the development of technology, the effects of parameter $\alpha$ on gravitational lensing may be detected in the future.

\section{SUMMARY}

Gravitational lensing in strong field limit provides a potentially powerful tool to identify the nature of black holes in the different gravity theories. In this paper we have investigated strong field lensing in the deformed Hořava-Lifshitz black hole spacetime. The model was applied to the supermassive black hole in the Galactic center. Our results show that with the increase of the parameter $\alpha$ the minimum impact parameter $u_{p s}$, the angular position of the relativistic images $\theta_{\infty}$ and the relative magnitudes $r_{m}$ decrease. The angular

separation $s$ increases. Comparing with the Reissner-Norström black hole, we find with the increase of parameter $\alpha$, the angular position $\theta_{\infty}$ decreases more slowly and $r_{m}$ more quickly, but angular separation $s$ increases more rapidly. This may offer a way to distinguish the deformed Hořava-Lifshitz black hole from a Reissner-Norström black hole by the astronomical instruments in the future.

\section{Acknowledgments}

This work was partially supported by the National Natural Science Foundation of China under Grant No.10875041; the Scientific Research Fund of Hunan Provincial Education Department Grant No.07B043 and the construct program of key disciplines in Hunan Province. J. L. Jing's work was partially supported by the National Natural Science Foundation of China under Grant No.10675045; the FANEDD under Grant No. 200317; and the Hunan Provincial Natural Science Foundation of China under Grant No.08JJ3010. 
[1] P. Schneider, J. Ehlers, and E. E. Falco, Gravitational Lenses, Springer-Verlag, Berlin (1992).

[2] A. F. Zakharov, Gravitational Lenses and Microlenses, Janus-K, Moscow (1997).

[3] R. D. Blandford and R. Narayan, Annu. Rev. Astron. Astrophys. 30, 311 (1992).

[4] C. Darwin, Proc. of the Royal Soc. of London 249, 180 (1959).

[5] K. S. Virbhadra, D. Narasimha and S. M. Chitre, Astron. Astrophys. 337 , 18 (1998).

[6] K. S. Virbhadra and G. F. R. Ellis, Phys. Rev. D 62, 084003 (2000).

[7] C. M. Claudel, K. S. Virbhadra and G. F. R. Ellis, J. Math. Phys. 42, 818 (2001).

[8] K. S. Virbhadra and G. F. R. Ellis, Phys. Rev.D 65, 103004(2002).

[9] S. Frittelly, T. P. Kling and E. T. Newman, Phys. Rev. D 61, 064021 (2000).

[10] V. Bozza, S. Capozziello, G. lovane and G. Scarpetta, Gen. Rel. and Grav. 33, 1535 (2001).

[11] E. F. Eiroa, G. E. Romero and D. F. Torres, Phys. Rev. D 66, 024010 (2002).

[12] E. F. Eiroa, Phys. Rev. D 71, 083010 (2005).

[13] R. Whisker, Phys. Rev. D 71, 064004 (2005).

[14] E. F. Eiroa, Phys. Rev. D 73, 043002 (2006).

[15] V. Bozza, Phys. Rev. D 66, 103001 (2002).

[16] V. Bozza, Phys. Rev. D 67, 103006 (2003);

[17] V. Bozza, F. De Luca, G. Scarpetta, and M. Sereno, Phys. Rev. D 72, 08300 (2005);

[18] V. Bozza, F. De Luca, and G. Scarpetta, Phys. Rev. D 74, 063001 (2006).

[19] A. Bhadra, Phys. Rev. D 67, 103009 (2003).

[20] K. Sarkar and A. Bhadra, Class. Quant. Grav. 23, 6101 (2006).

[21] R. A. Konoplya, Phys. Rev. D 74, 124015 (2006); Phys. Lett. B 644, 219 (2007).

[22] N. Mukherjee and A. S. Majumdar, astro-ph/0605224 (2006).

[23] V. Perlick, Phys. Rev. D 69, 064017 (2004).

[24] G. N. Gyulchev and S. S. Yazadjiev, gr-qc/0611110

[25] P. Hořava, Phys. Rev. D 79 (2009) 084008 arXiv:0901.3775 [hep-th]].

[26] P. Hořava, JHEP 0903 (2009) 020 arXiv:0812.4287 [hep-th]].

[27] P. Hořava, arXiv:0902.3657 [hep-th].

[28] A. Volovich and C. Wen, arXiv: 0903.2455 [hep-th].

[29] J. Kluson, arXiv:0904.1343 [hep-th].

[30] H. Nikolic, arXiv:0904.3412 [hep-th].

[31] H. Nastase, arXiv:0904.3604 [hep-th].

[32] K. I. Izawa, arXiv:0904.3593 [hep-th].

[33] G. E. Volovik, arXiv:0904.4113 [gr-qc].

[34] B. Chen and Q. G. Huang, arXiv:0904.4565 [hep-th].

[35] R. G. Cai, B. Hu and H. B. Zhang, arXiv:0905.0255 [hep-th].

[36] G. Calcagni, arXiv:0904.0829 [hep-th]. 
[37] T. Takahashi and J. Soda, arXiv:0904.0554 [hep-th].

[38] S. Mukohyama, arXiv:0904.2190 [hep-th].

[39] R. Brandenberger, arXiv:0904.2835 [hep-th].

[40] Y. S. Piao, arXiv:0904.4117 [hep-th].

[41] X. Gao, arXiv:0904.4187 [hep-th].

[42] E. Kiritsis and G. Kofinas, arXiv:0904.1334 [hep-th].

[43] E. O. Colgain and H. Yavartanoo, arXiv:0904.4357 [hep-th].

[44] A. Kehagias and K. Sfetsos, arXiv:0905.0477 [hep-th].

[45] H. Lu, J. Mei and C. N. Pope, arXiv:0904.1595 [hep-th].

[46] R. G. Cai, L. M. Cao and N. Ohta, arXiv:0904.3670 [hep-th].

[47] R. G. Cai, Y. Liu and Y. W. Sun, arXiv:0904.4104 [hep-th].

[48] A. Ghodsi, arXiv:0905.0836 [hep-th].

[49] Y. S. Myung and Y. W. Kim, arXiv:0905.0179 [hep-th].

[50] T. Nishioka, arXiv:0905.0473 [hep-th].

[51] R. G. Cai, L. M. Cao and N. Ohta, arXiv:0905.0751 [hep-th].

[52] Y. S. Myung, arXiv:0905.0957 [gr-qc]].

[53] S. B. Chen and J. L. Jing, arXiv: 0905.1409.

[54] R. A. Konoplya. arXiv: 0905.1523. 